\title{
INVARIANT SUBSPACES FOR ALGEBRAS OF LINEAR OPERATORS AND AMENABLE LOCALLY COMPACT GROUPS
}

\author{
ANTHONY T. M. LAU AND JAMES C. S. WONG \\ (Communicated by Richard R. Goldberg)
}

\begin{abstract}
Let $G$ be a locally compact group. We prove in this paper that $G$ is amenable if and only if the group algebra $L_{1}(G)$ (respectively the measure algebra $M(G)$ ) satisfies a finite-dimensional invariant subspace property $T(n)$ for $n$-dimensional subspaces contained in a subset $X$ of a separated locally convex space $E$ when $L_{1}(G)$ (respectively $M(G)$ ) is represented as continuous linear operators on $E$. We also prove that for any locally compact group, the Fourier algebra $A(G)$ and the Fourier Stieltjes algebra $B(G)$ always satisfy $T(n)$ for each $n=1,2, \ldots$.
\end{abstract}

1. Introduction. Let $E$ be a separated locally convex space and $X$ a subset of $E$ containing an $n$-dimensional subspace. In [4], K. Fan obtained the following finite-dimensional invariant subspace property $P(n)$ for $n$-dimensional subspaces contained in $X$ : If $S=\left\{T_{s}: s \in S\right\}$ is a representation of a left amenable (discrete) semigroup $S$ as continuous linear operators from $E$ into $E$ such that $T_{s}(L)$ is an $n$-dimensional subspace contained in $X$ whenever $L$ is one and $s \in S$, and there exists a closed $S$-invariant subspace $H$ in $E$ of codimension $n$ with the property that $(x+H) \cap X$ is compact convex for each $x \in E$, then there exists an $n$-dimensional subspace $L_{0}$ contained in $X$ such that $T_{s}\left(L_{0}\right)=L_{0}$ for all $s \in S$. Conversely, as proved by Lau [10] (see also [12]), if $S$ satisfies $P(1)$, then $S$ is left amenable.

In this paper, we prove among other things that (Theorem 3.5) if $G$ is an amenable locally compact group, then the group algebra $L_{1}(G)$ and measure algebra $M(G)$ satisfy a similar finite-dimensional invariant subspace property $T(n)$ for $n$-dimensional subspaces contained in a subset $X$ of a separated locally convex subspace $E$ when $L_{1}(G)$ (or $M(G)$ ) is represented as continuous linear operators on $E$. Conversely, if $L_{1}(G)$ or $M(G)$ satisfies $T(1)$, then $G$ is amenable. We also show that (Theorem 3.6) if $G$ in any locally compact group, then both the Fourier algebra $A(G)$ and Fourier Stieltjes algebra $B(G)$ (see Eymard [3]) always satisfy $T(n)$ for each $n=1,2, \ldots$.

For convenience and to avoid repetition of arguments, we find it most natural to phrase our main result (Theorem 3.4) in terms of $F$-algebras (see [11]). Definition and information on $F$-algebras needed for the proof of our main result will be gathered in $\S 2$.

Received by the editors September 22, 1986. Presented to the Canadian Mathematical Society 1986 Winter Meeting in Ottawa, Canada.

1980 Mathematics Subject Classification (1985 Revision). Primary 43A07; Secondary 43A010, 43A20, 43A30.

Key words and phrases. Amenable locally compact groups, group algebra, measure algebra, Fourier algebra, Fourier Stieltjes algebra, finite-dimensional, invariant subspaces. 
2. $F$-algebras. By an $F$-algebra we shall mean a complex Banach algebra $A$ such that $A^{*}$ is a $C^{*}$-algebra and the identity of $A^{*}$, denoted by $I$ (which always exists [15, Proposition 1.6.1]), is a multiplicative linear functional on $A$. Examples of $F$-algebras include the group algebra $L_{1}(G)$, Fourier algebra $A(G)$ and the Fourier Stieltjes algebra $B(G)$ of a locally compact group $G$ (see [11] for details). It also includes the measure algebra $M(S)$ of a locally compact semigroup $S$.

Let $S_{A}$ denote the set of all positive functionals in $A \subset A^{* *}$ with norm one. Then $S_{A}=\{\mu \in A ;\|\mu\|=I(\mu)=1\}\left[15\right.$, p. 9]. Hence, as readily checked, $\left(S_{A}, *\right)$, where $*$ denotes the multiplication of $A$, is a semigroup. $A$ is called left amenable if $A^{*}=M$ has a topological left invariant mean (abbreviated as TLIM), i.e. an $m \in M^{*}$ such that $\|m\|=1, m \geq 0$ and $m(F \cdot \mu)=m(F)$ for each $\mu \in S_{A}$ and $F \in M$, where $F \cdot \mu \in M$ is defined by $\langle F \cdot \mu, \nu\rangle=\langle F, \mu * \nu\rangle$ for all $\nu \in A$.

A function $f \in \operatorname{CB}\left(S_{A}\right)$ (the continuous bounded functions on $S_{A}$ ), is called additively uniformly continuous on $S_{A}$ if given $\varepsilon>0$, there is some $\delta>0$ such that $\mu, \nu \in S_{A}$ and $\|\mu-\nu\|<\varepsilon$ implies $|f(\mu)-f(\nu)|<\varepsilon$. Let $\operatorname{AUC}\left(S_{A}\right)$ denote the space of all such functions. It is straightforward to show that $\operatorname{AUC}\left(S_{A}\right)$ is a norm closed translation invariant subspace of $\mathrm{CB}\left(S_{A}\right)$ containing constants and restrictions of elements in $A^{*}$ to $S_{A}$. A linear functional $m \in \operatorname{AUC}\left(S_{A}\right)^{*}$ is called a left invariant mean if $\|m\|=m(1)=1$ and $m\left(l_{\mu} f\right)=m(f), \forall f \in \operatorname{AUC}\left(S_{A}\right), \mu \in S_{A}$, where $\left(l_{\mu} f\right)(\nu)=f(\mu * \nu)$ for all $\nu \in S_{A}$. The following lemma, which we shall need, is due to Ganeson [5] for the case $A=L_{1}(G)$ of a locally compact group. Because part of his proof depends heavily on the locally compact group structure, we include a simple proof here for completeness.

LEMMA 2.1. The following statements are equivalent for an $F$-algebra $A$ :

(a) $A$ is left amenable.

(b) $\operatorname{AUC}\left(S_{A}\right)$ has a left invariant mean.

ProOF. (a) implies (b). By [11, Theorem 4.6], there is a net $\mu_{\alpha} \in S_{A}$ such that $\mu * \mu_{\alpha}-\mu_{\alpha} \rightarrow 0$ in the norm topology for each $\mu \in S_{A}$. For each $\alpha$, define $m_{\alpha}(f)=f\left(\mu_{\alpha}\right)$ for each $f \in \operatorname{AUC}\left(S_{A}\right)$. Let $m$ be a weak*-cluster point of $\left\{m_{\alpha}\right\}$. Then $m$ is a left invariant mean on $\operatorname{AUC}\left(S_{A}\right)$.

(b) implies (a). Define $\tau: A^{*} \rightarrow \mathrm{CB}\left(S_{A}\right)$ by $\tau(F)(\mu)=F(\mu), F \in A^{*}, \mu \in S_{A}$. Then $\tau$ is a continuous linear map of $A^{*}$ into $\operatorname{CB}\left(S_{A}\right)$ such that $\tau \geq 0, \tau(1)=1$ and $\tau(F \cdot \mu)=l_{\mu}(\tau(F))$ where $\mu \in S_{A}, F \in A^{*}$ and $l_{\mu}$ is the left translation operator in $\mathrm{CB}\left(S_{A}\right)$. Moreover, $\tau(F) \in \operatorname{AUC}\left(S_{A}\right)$. Hence $\tau^{*}: \operatorname{AUC}\left(S_{A}\right)^{*} \rightarrow A^{* *}$. If $m$ is a left invariant mean on $\operatorname{AUC}\left(S_{A}\right)$, then $\tau^{*}(m)$ is a TLIM on $A^{*}$.

3. Algebra of operators. A representation of an $F$-algebra $A$ as operators in a locally convex space $E$ is a map $T: A \times E \rightarrow E$ denoted by $(\mu, x) \rightarrow T_{\mu}(x)$ such that (1) $T_{\mu}: E \rightarrow E$ is continuous and linear, (2) $\mu \rightarrow T_{\mu}(x)$ is continuous and linear with respect to the norm topology in $A$ for each $x \in E$ and (3) $T_{\mu * \nu}=$ $T_{\mu} \circ T_{\nu} \forall \mu, \nu \in A$, where * denotes multiplication in $A$. Also, let $X$ be a subset of $E$ containing an $n$-dimensional subspace. As in Lau $[\mathbf{1 0}], \mathcal{L}_{n}(X)$ denotes all $n$-dimensional subspaces of $E$ contained in $X$. We say that $\mathcal{L}_{n}(X)$ is $S_{A}$-invariant under $T$ if $T_{\mu}(L) \in \mathcal{L}_{n}(X)$ for each $L \in \mathcal{L}_{n}(X)$ and $\mu \in S_{A}$. A closed subspace $H$ in $E$ is called $S_{A}$-invariant under $T$ if $T_{\mu}(H) \subset H \forall \mu \in S_{A}$ (and hence $\forall \mu \in A$ as well). Denote by $q: E \rightarrow E / H$ the natural map such that $q(x)=\tilde{x}, x \in E$. 
LemMA 3.1. Let $T: A \times E \rightarrow E$ be a representation of an $F$-algebra $A$ and $H$ a closed $S_{A}$-invariant subspace of $E$ of codimension $n$. Also let $X$ be a subset of $E$ such that $(x+H) \cap X$ is compact and convex for each $x \in E$. Denote by $\mathcal{K}$ the set of all linear maps $B \in \mathcal{L}(F, E)$ such that $B(y) \in q^{-1}(y) \cap X \forall y \in F$. If $\mathcal{L}_{n}(X)$ is nonempty and $S_{A}$-invariant, then for each $\mu \in A$, the map $T_{\mu}: E \rightarrow E$ induces a map $\tilde{T}_{\mu}: F \rightarrow F$ where $F=E / H$ such that $q \circ T_{\mu}=\tilde{T}_{\mu} \circ q \forall \mu \in A$. Moreover, if $\mu \in S_{A}, \tilde{T}_{\mu}$ is an isomorphism of $F$ onto itself and $\mathcal{K} \neq \varnothing$ is convex and compact in the separated locally convex space $\mathcal{L}(F, E)$ with the topology $\tau$ of pointwise convergence. Defining $\psi: S_{A} \times \mathcal{K} \rightarrow \mathcal{K}$ by $\psi_{\mu}(B)=T_{\mu} \circ B \circ \tilde{T}_{\mu}^{-1}$, $\mu \in S_{A}, B \in \mathcal{K}$, then $\psi$ is an affine action of the semigroup $S_{A}$ on $\mathcal{K}$.

ProOF. The proof is basically contained in Fan [4, Theorem, p. 447]. We need only consider the semigroup $S_{A}$ of linear operators in $E$. Note that $\tilde{T}_{\mu}$ is defined for $\mu \in A$ (not just in $S_{A}$ ). However $\tilde{T}_{\mu}$ need not be an isomorphism unless $\mu \in S_{A}$.

DEFINITION 3.2. The action of $S_{A}$ on $E$ is called inversely equicontinuous modulo $H$ if given any neighborhood $U$ in $E$, there is some neighborhood $V$ in $E$ such that $V \subset T_{\mu}(U)+H$ for any $\mu \in S_{A}$. This is equivalent to the condition that the family $\left\{\tilde{T}_{\mu}^{-1}: \mu \in S_{A}\right\}$ is equicontinuous on $F$.

LEMMA 3.3. Under the hypothesis of Lemma 3.1 and if $A$ is left amenable and the action of $S_{A}$ on $E$ is inversely equicontinuous modulo $H$, then the induced action $\tilde{T}: S_{A} \times F \rightarrow F$, where $(\mu, y) \rightarrow \tilde{T}_{\mu} y, \mu \in S_{A}$ and $y \in F$, is similar to a unitary representation of the semigroup $S_{A}$ on the Hilbert space $F$. Moreover, the representation functions $\mu \rightarrow\left(\tilde{T}_{\mu} y, z\right), y, z \in F$, are in $\operatorname{AUC}\left(S_{A}\right)$. The same is ture for the inverse (anti) representation $\left\{\tilde{T}_{\mu}^{-1}: \mu \in S_{A}\right\}$.

ProOF. Fix a basis $\left\{\tilde{e}_{1}, \ldots, \tilde{e}_{n}\right\}$ in $F$ and define $(y, z)=\sum_{j=1}^{n} \alpha_{j} \bar{\beta}_{j}$ where $y=$ $\sum_{j=1}^{n} \alpha_{j} \tilde{e}_{j}$ and $z=\sum_{j=1}^{n} \beta_{j} \tilde{e}_{j}$ are in $F$, and let $\|\cdot\|$ denote the induced (Euclidean) norm. Consider the adjoint (anti)representation of $S_{A}$ on $F$, i.e. $\tilde{T}^{*}: S_{A} \times F \rightarrow F$ such that $(\mu, y) \rightarrow \tilde{T}_{\mu}^{*} y$ where $\mu \in S_{A}, y \in F$ and $\tilde{T}_{\mu}^{*}$ is the adjoint of $\tilde{T}_{\mu}$ on the Hilbert space $F$. Since the map $\mu \rightarrow \tilde{T}_{\mu}^{*} y$ is continuous and conjugate linear on $A$ and since

$$
\left|\left\|\tilde{T}_{\mu}^{*} y\right\|-\left\|\tilde{T}_{\nu}^{*} y\right\|\right| \leq\left\|\tilde{T}_{\mu}^{*} y-\tilde{T}_{\nu}^{*} y\right\| \leq\|\mu-\nu\| \cdot M(y) \quad \forall \mu, \nu \in S_{A},
$$

where $M(y) \geq 0$, it follows that the function $\mu \rightarrow\left\|\tilde{T}_{\mu}^{*} y\right\|$ and hence $\mu \rightarrow\left\|\tilde{T}_{\mu}^{*} y\right\|^{2}$ on $S_{A}$ is in $\operatorname{AUC}\left(S_{A}\right)$. By the Polarisation Principle, the function $\mu \rightarrow\left(\tilde{T}_{\mu}^{*} y, \tilde{T}_{\mu}^{*} z\right)$ (restricted to $S_{A}$ ) is also in $\operatorname{AUC}\left(S_{A}\right)$. Let $m$ be a left invariant mean on $\operatorname{AUC}\left(S_{A}\right)$ (by Lemma 2.1). Define a new inner product on $F$ by $[y, z]=\left\langle m(\mu),\left(\tilde{T}_{\mu}^{*} y, \tilde{T}_{\mu}^{*} z\right)\right\rangle$. Now the family $\left\{\tilde{T}_{\mu}^{*}: \mu \in S_{A}\right\}$ is pointwise bounded on $F$, hence bounded on $F$ (by the Principle of Uniform Boundedness). On the other hand, inverse equicontinuity of $S_{A}$ modulo $H$ implies the family $\left\{\tilde{T}_{\mu}^{-1}: \mu \in S_{A}\right\}$ is equicontinuous hence uniformly bounded on $F$. So is the family $\left\{\left(\tilde{T}_{\mu}^{*}\right)^{-1}: \mu \in S_{A}\right\}=\left\{\left(\tilde{T}_{\mu}^{-1}\right)^{*}: \mu \in S_{A}\right\}$. Therefore there exist $M, N>0$ such that

$$
\begin{aligned}
N^{2}\|y\|^{2} & \leq \inf \left\{\left\|\tilde{T}_{\mu}^{*}(y)\right\|^{2}: \mu \in S_{A}\right\} \leq\left\langle m(\mu),\left\|\tilde{T}_{\mu}^{*}(y)\right\|^{2}\right\rangle \\
& =|y|^{2} \leq \sup \left\{\left\|\tilde{T}_{\mu}^{*}(y)\right\|^{2}: \mu \in S_{A}\right\} \leq M^{2}\|y\|^{2},
\end{aligned}
$$


where $|y|^{2}=[y, y]$. Consequently, the two norms are equivalent and hence, as is well known (see [7]), there is an invertible bicontinuous selfadjoint linear operator $Q \in B(F)$ such that $[y, z]=(Q y, Q z) \forall y, z \in F$. Now $\forall \mu \in S_{A}$,

$$
\begin{aligned}
\left\|Q \tilde{T}_{\mu}^{*} Q^{-1}(y)\right\|^{2} & =\left|\tilde{T}_{\mu}^{*} Q^{-1}(y)\right|^{2}=\left\langle m(\nu),\left\|\tilde{T}_{\nu}^{*} \tilde{T}_{\mu}^{*} Q^{-1}(y)\right\|^{2}\right\rangle \\
& \left.=\left\langle m(\nu), \| \tilde{T}_{\mu * \nu}^{*} Q^{-1} y\right) \|^{2}\right\rangle=\left\langle m(\nu),\left\|\tilde{T}_{\nu}^{*} Q^{-1}(y)\right\|^{2}\right\rangle \\
& =\left|Q^{-1}(y)\right|^{2}=\|y\|^{2}
\end{aligned}
$$

by left invariance of $m$. Therefore $U_{\mu}=Q \tilde{T}_{\mu}^{*} Q^{-1}$ is unitary for any $\mu \in S_{A}$. This implies that $\left\{\tilde{T}_{\mu}: \mu \in S_{A}\right\}$ is similar to a unitary representation on $F$. Finally, since $\mu \rightarrow\left(\tilde{T}_{\mu} y, z\right)$ is bounded linear on $A$, its restriction to $S_{A}$ is in $\operatorname{AUC}\left(S_{A}\right)$. To show that the same is true for the inverse representation, we first observe that since $F$ is finite dimensional, the strong operator topology and the uniform operator topology agree on $B(F)$. Hence $\mu \rightarrow \tilde{T}_{\mu}^{*}$ is also continuous when $B(F)$ has the uniform operator topology. Consequently given $\varepsilon>0$, there exists $\delta>0$ such that $\mu, \nu \in A$, $\|\mu-\nu\|<\delta$ implies $\left\|\tilde{T}_{\mu}^{*}-\tilde{T}_{\nu}^{*}\right\|<\varepsilon$. In particular, if $\mu, \nu \in S_{A}$ and $\|\mu-\nu\|<\delta$, then $\left\|U_{\mu}-U_{\nu}\right\|=\left\|Q \tilde{T}_{\mu}^{*} Q^{-1}-Q \tilde{T}_{\nu}^{*} Q^{-1}\right\| \leq\|Q\| \cdot\left\|Q^{-1}\right\| \cdot \varepsilon$. But $\tilde{T}_{\mu}^{-1}=Q U_{\mu} Q^{-1}$ $\forall \mu \in S_{A}$, since $U_{\mu}$ is unitary for such $\mu$ and $Q$ is selfadjoint. Therefore

$$
\left\|\tilde{T}_{\mu}^{-1}-\tilde{T}_{\nu}^{-1}\right\| \leq\|Q\|^{2} \cdot\left\|Q^{-1}\right\|^{2} \cdot \varepsilon \quad \forall \mu, \nu \in S_{A}
$$

with $\|\mu-\nu\|<\delta$. This implies that the function $\mu \rightarrow y^{*} \tilde{T}_{\mu}^{-1} y$ on $S_{A}$ is in $\operatorname{AUC}\left(S_{A}\right)$ $\forall y \in F, y^{*} \in F^{*}$.

THEOREM 3.4. Let $A$ be an $F$-algebra.

(a) If $A$ is left amenable, then $A$ satisfies property $T(n)$ for $n=1,2, \ldots$ where property $T(n)$ is defined as follows: Let $E$ be a separated locally convex space and $T: A \times E \rightarrow E$ be a representation of $A$ as linear operators in $E$. Let $X$ be a subset of $E$ such that there exists a closed $S_{A}$-invariant subspace $H$ of $E$ of codimension $n$ and $(x+H) \cap X$ is compact convex for each $x \in E$. If the action of $S_{A}$ on $E$ is inversely equicontinuous modulo $H$ and $\mathcal{L}_{n}(X)$ is nonempty and $S_{A}$-invariant, then there exists $L_{0} \in \mathcal{L}_{n}(X)$ such that $T_{\mu}\left(L_{0}\right)=L_{0} \forall \mu \in S_{A}$.

(b) If $S$ satisfies property $T(1)$, then $A$ is left amenable (hence $A$ satisfies $T(n)$ for every $n$ ).

PROOF. (a) With the notation of Lemma 3.1, define the affine representaion $\Psi: S_{1} \times \mathcal{K} \rightarrow \mathcal{K}$ by $\Psi_{\mu}(B)=T_{\mu} \circ B \circ \tilde{T}_{\mu}^{-1}, \mu \in S_{A}$ and $B \in \mathcal{K}$. We want to show that $\psi$ is an $A$-representation for the pair $S_{A}$ and $\operatorname{AUC}\left(S_{A}\right)$ in the sense of Argabright [1, $\S 2]$. That is, for each $h \in A(\mathcal{K})$, the affine continuous functions on $\mathcal{K}$, the function $\mu \rightarrow h\left(\Psi_{\mu}(B)\right)$ is in $\operatorname{AUC}\left(S_{A}\right)$ for each $B \in \mathcal{K}$. By Argabright [1, Lemma 1] and Kelley and Namioka [9, Theorem 14.6, p. 120], it is enought to consider $h \in A(K)$ of the form $h(B)=x^{*} B y, B \in \mathcal{K}$, where $x^{*} \in E^{*}$ and $y \in F$. Let $\left\{\tilde{e}_{1}, \ldots, \tilde{e}_{n}\right\}$ be a basis in $F$. Then $\left\{q T_{\mu} B \tilde{e}_{1}, \ldots, q T_{\mu} B \tilde{e}_{n}\right\}$ is also a basis in $F$ for any $\mu \in S_{A}$ and $B \in \mathcal{K}$ (by definition of $\mathcal{K}, S_{A}$-invariance of $\mathcal{L}_{n}(X)$ and the fact that $(x+H) \cap X$ is compact and convex for each $x \in E)$. Write $y=\sum_{j=1}^{n} \alpha_{j}(\mu) q T_{\mu} B\left(\tilde{e}_{j}\right)$ where $\mu \in S_{A}, B \in \mathcal{K}$. Note that the scalars $\alpha_{j}(\mu)$ depend only on $\mu \in S_{A}$ and not on $B$. (See Fan [4, equation (7), p. 449].) Then $\tilde{T}_{\mu}^{-1}(y)=\sum_{j=1}^{n} \alpha_{j}(\mu) \tilde{e}_{j}, \mu \in S_{A}$. (Since $q \circ B=$ identity on $F$.) By Lemma 3.3, the functions $\mu \rightarrow \alpha_{k}(\mu)=y_{k}^{*} \tilde{T}_{\mu}^{-1}(y)$ on 
$S_{A}$ belong to $\operatorname{AUC}\left(S_{A}\right)$ where $\left\{y_{1}^{*}, \ldots, y_{n}^{*}\right\}$ is the basis dual to $\left\{\tilde{e}_{1}, \ldots, \tilde{e}_{n}\right\}$. Now

$$
h\left(\Psi_{\mu}(B)\right)=x^{*}\left(T_{\mu} \circ B \circ \tilde{T}_{\mu}^{-1}\right) y=\sum_{j=1}^{n} \alpha_{j}(\mu) x^{*} T_{\mu} B\left(\tilde{e}_{j}\right), \quad \mu \in S_{A} .
$$

Therefore the function $\mu \rightarrow h\left(\Psi_{\mu}(B)\right)$ on $S_{A}$ is in $\operatorname{AUC}\left(S_{A}\right)$. Hence $\Psi: S_{1} \times \mathcal{K} \rightarrow \mathcal{K}$ is an $A$-representation for the pair $S_{1}, \operatorname{AUC}\left(S_{A}\right)$. By Argabright [1, Theorem 1], $\Psi$ has a common fixed point $Q_{0} \in \mathcal{K}$. Put $L_{0}=Q_{0}(F) \in \mathcal{L}_{n}(X)$, then $T_{\mu}\left(L_{0}\right)=L_{0}$ $\forall \mu \in S_{A}$.

(b) Define $E=A^{* *}$ with the weak* topology and $T: A \times E \rightarrow E$ by $T_{\mu}(m)=l_{\mu}^{*} m$ where $l_{\mu}^{*}$ is the adjoint of the map $l_{\mu}: A^{*} \rightarrow A^{*}$ such that $l_{\mu}(F)(\nu)=F(\mu * \nu)$, $\nu \in A$. As in Lau [10], let $X$ be the union of all one-dimensional subspaces of $E=A^{* *}$ generated by the means (states) on $A^{*}$ and $H=\left\{m \in A^{* *}: m(I)=0\right\}$. The arguments used in the proof of Lau [7, Theorem 1(b)] show that the hypotheses in property $T(1)$ are all satisfied except the part on inverse equicontinuity modulo $H$ of $S_{A}$, which is also satisfied because the induced action $\tilde{T}_{\mu}$ is independent of $\mu \in S_{A} .\left(l_{\mu}(I)=I\right.$ if $\mu \in S_{A}$, since $I(\mu)=1$, if $\mu \in S_{A}$.) Since $S$ satisfies $T(1)$, this means $l_{\mu}^{*}\left(L_{0}\right)=L_{0} \forall \mu \in S_{A}$, where $L_{0}$ is a one-dimensional subspace generated by some mean $m_{0}$. Necessarily, $m_{0}$ is a topological left invariant mean on $A^{*}$.

A locally compact group $G$ is amenable if the space $\operatorname{CB}(G)$ has a left invariant mean. Examples of amenable locally compact groups include all solvable groups, abelian groups and all compact groups. However, if $G$ contains the free group on two generators as a closed subgroup, then $G$ is not amenable (see Greenleaf [7] or Pier [13] for details).

THEOREM 3.5. Let $G$ be a locally compact group. If $G$ is amenable, then the group algebra $L_{1}(G)$ and the measure algebra $M(G)$ satisfy $T(n)$ for each $n=$ $1,2,3, \ldots$ Conversely, if either $L_{1}(G)$ or $M(G)$ satisfies $T(1)$, then $G$ is amenable.

ProOF. It follows from Greenleaf [7, Theorem 2.2.1] and Wong [17, Theorem 3.3] that amenability of $G$ is equivalent to the left amenability of $L_{1}(G)$ and $M(G)$.

THEOREM 3.6. Let $G$ be a locally compact group. Then both the Fourier algebra $A(G)$ and the Fourier Stieltjes algebra $B(G)$ have property $T(n)$ for each $n=$ $1,2, \ldots$

Proof. In this case, if $A=A(G)$ or $B(G)$, the $A$ is a commutative $F$-algebra (see Eymard [3]). Hence $S_{A}$ is commutative and so $l_{\infty}\left(S_{A}\right)$, the space of bounded complex-valued functions on the discrete semigroup $S_{A}$, has an invariant mean (see Day [2]). So by Lemma 2.1, $A$ is left amenable. Now apply Theorem 3.4.

Given a semigroup $S$, let $l_{1}(S)$ be the Banach algebra as defined in Day [2] or Hewitt and Zuckermann [8]. $S$ is called left amenable if $l_{\infty}(S)$ has a left invariant mean (see Day [2]).

THEOREM 3.7. Let $S$ be a semigroup. If $S$ is left amenable, then the Banach algebra $l_{1}(S)$ satisfies $T(n)$ for each $n=1,2,3, \ldots$ Conversely if $l_{1}(S)$ satisfies $T(1)$, then $S$ is left amenable.

PROOF. Any left invariant mean on $l_{\infty}(S)$ is necessarily topological left invariant. Now apply Theorem 3.4 again. 
REMARKS. (a) Left amenability of the Fourier algebra $A(G)$ of a locally compact group $G$ was proved by Renauld [14, Theorem 4] (see also Granirer [6, Proposition $5(\mathrm{a})])$ by a completely different method.

(b) Theorem 3.7 can be generalized to all locally compact semigroups $S$ with left amenability of $S$ replaced by topological left amenability of the measure algebra $M(S)$ (see [16]), and $l_{1}(S)$ replaced by $M(S)$.

\section{REFERENCES}

1. L. N. Argabright, Invariant means and fixed points, a sequel to Mitchell's paper, Trans. Amer. Math. Soc. 130 (1968), 127-130.

2. M. M. Day, Amenable semigroups, Illinois J. Math. 1 (1957), 509-544.

3. P. Eymard, L'algèbre de Fourier d'un groupe localement compact, Bull. Soc. Math. France 92 (1964), 181-236.

4. K. Fan, Invariant subspaces for a semigroup of linear operators, Indag. Math. 27 (1965), 447451.

5. S. Ganeson, On amenability of the semigroup of probability measures on topological groups, Ph.D. thesis, SUNY at Albany, 1983.

6. E. E. Granirer, Weakly almost periodic and uniformly continuous functionals on the Fourier algebra of any locally compact groups, Trans. Amer. Math. Soc. 189 (1974), 371-381.

7. F. P. Greenleaf, Invariant means on topological groups, Van Nostrand Math. Studies, Van Nostrand, Princeton, N.J., 1969.

8. E. Hewitt and H. S. Zuckerman, The $l_{1}$-algebra of a commutative semigroup, Trans. Amer. Math. Soc. 83 (1956), 70-97.

9. J. Kelley and I. Namioka, Linear topological spaces, Van Nostrand, Princeton, N.J., 1963.

10. A. Lau, Finite dimensional invariant subspaces for a semigroup of linear operators, J. Math. Anal. App. 97 (1983), 374-379.

11. __ Analysis on a class of Banach algebras with applications to harmonic analysis on locally compact groups and semigroups, Fund. Math. 118 (1983), 161-175.

12. A. Lau and James C. S. Wong, Finite dimensional invariant subspaces for measurable semigroups of linear operators, J. Math. Anal. Appl. 127 (1987), 548-558.

13. J. P. Pier, Amenable locally compact groups, Wiley, 1984.

14. P. F. Renaud, Invariant means on a class of von Neumann algebras, Trans. Amer. Math. Soc. 170 (1972), 285-291.

15. S. Sakai, $C^{*}$-algebras and $W^{*}$-algebras, Springer-Verlag, 1971.

16. James C. S. Wong, An ergodic property of a locally compact amenable semigroup, Pacific J. Math. 48 (1973), 615-619.

17. Soc. 27 (1971), 572-578.

Department of Mathematics, The University of Alberta, Edmonton, AlBERTA, CANADA T6G 2G1

Department of Mathematics and Statistics, The University of Calgary, Calgary, Alberta, Canada T2N 1N4 